\title{
Supplementary Info
}

\section{Reproducing springtime Arctic tropospheric ozone depletion events in an outdoor mesocosm sea-ice facility}

5 Zhiyuan $\mathrm{Gao}^{1}$, Nicolas-Xavier Geilfus ${ }^{1}$, Alfonso Saiz-Lopez ${ }^{2}$, and Feiyue Wang ${ }^{1}$

${ }^{1}$ Centre for Earth Observation Science, and Department of Environment and Geography, University of Manitoba, Winnipeg, MB R3T 2N2, Canada

${ }^{2}$ Department of Atmospheric Chemistry and Climate, Institute of Physical Chemistry Rocasolano, CSIC, 28006 Madrid, Spain

10 Correspondence: Feiyue Wang (feiyue.wang@umanitoba.ca)

This supplementary info contains two figures:

15 Figure S1. Light transmittance of the two acrylic tubes used in the experiments

Figure S2. Temporal changes of wind speed measured at $1.5 \mathrm{~m}$ above the ice surface 


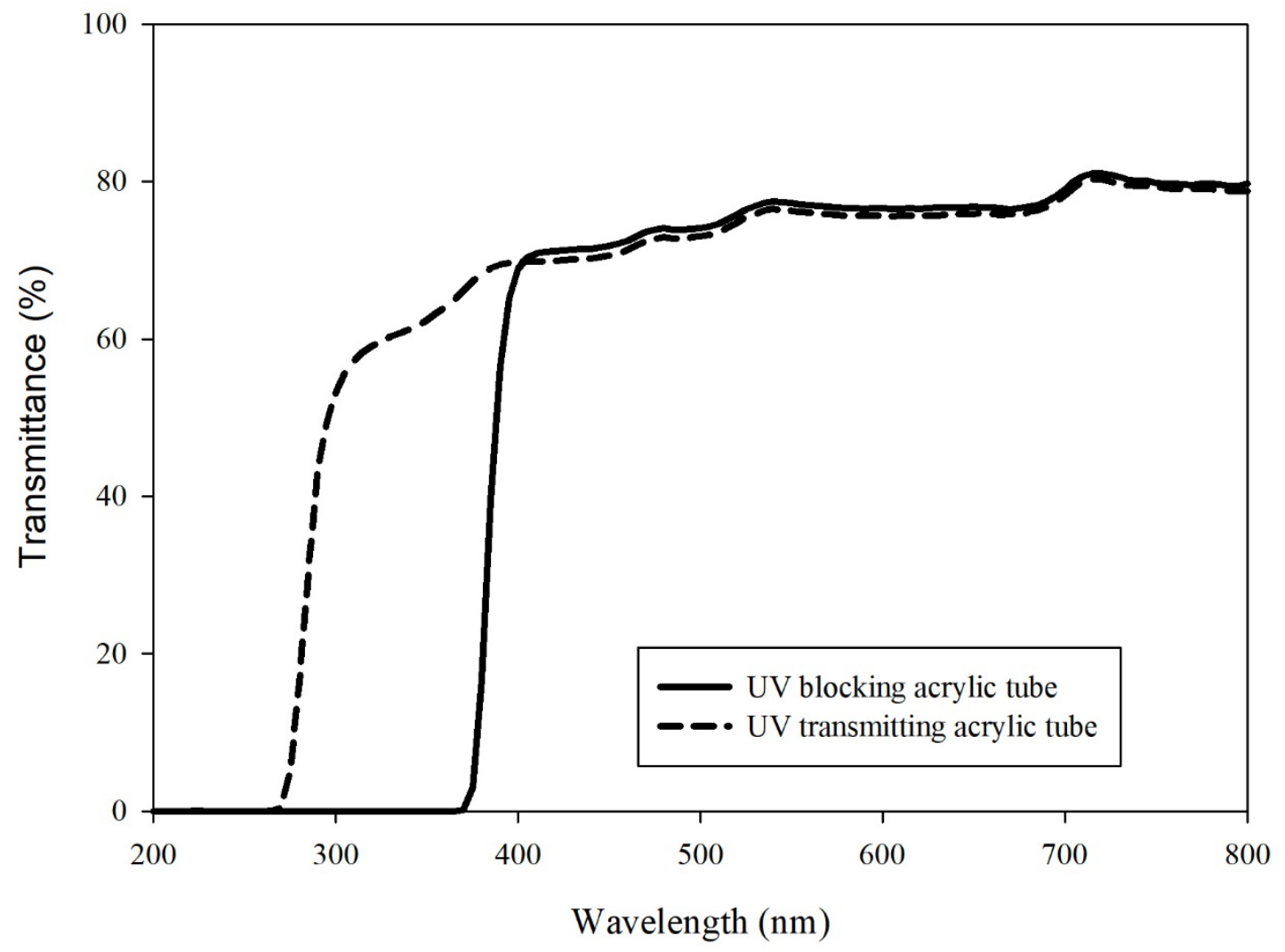

20 Figure S1. Light transmittance of the two acrylic tubes used in the experiments

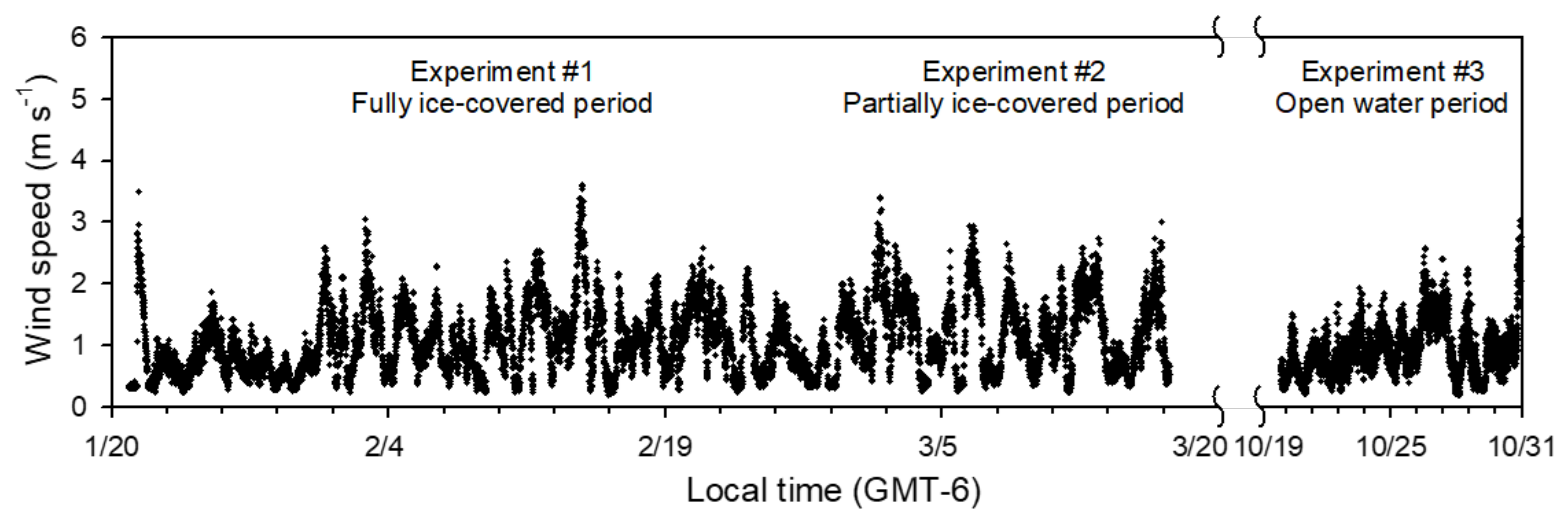

Figure S2. Temporal changes of wind speed measured at $1.5 \mathrm{~m}$ above the ice surface 